Research Article

\title{
Identification of the Active Constituents and Significant Pathways of Cangfu Daotan Decoction for the Treatment of PCOS Based on Network Pharmacology
}

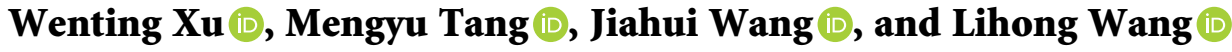 \\ Department of Reproduction, Zhangjiagang TCM Hospital Affiliated to Nanjing University of Chinese Medicine, Zhangjiagang, \\ Suzhou, Jiangsu, China \\ Correspondence should be addressed to Lihong Wang; zjgzywlh@njucm.edu.cn
}

Received 18 December 2019; Accepted 27 January 2020; Published 22 February 2020

Academic Editor: Deborah A. Kennedy

Copyright (C) 2020 Wenting Xu et al. This is an open access article distributed under the Creative Commons Attribution License, which permits unrestricted use, distribution, and reproduction in any medium, provided the original work is properly cited.

\begin{abstract}
Background. Polycystic ovary syndrome (PCOS) is the most common female endocrine disease. Cangfu Daotan Decoction (CDD) can effectively relieve the clinical symptoms of PCOS patients. Methods. To explore the active ingredients and related pathways of CDD for treating PCOS, a network pharmacology-based analysis was carried out. The active ingredients of CDD and their potential targets were obtained from the TCM system pharmacology analysis platform. The obtained PCOS-related genes from OMIM and GeneCards were imported to establish protein-protein interaction networks in STRING. Finally, GO analysis and significant pathway analysis were conducted with the RStudio (Bioconductor) database. Results. A total of 111 active compounds were obtained from 1433 ingredients present in the CDD, related to 118 protein targets. In addition, 736 genes were found to be closely related to PCOS, of which 44 overlapped with CDD and were thus considered therapeutically relevant. Pathway enrichment analysis identified the AGE-RAGE signalling pathway in diabetic complications, endocrine resistance, the IL-17 signalling pathway, the prolactin signalling pathway, and the HIF-1 signalling pathway. Moreover, PI3K-Akt, insulin resistance, Toll-like receptor, MAPK, and AGE-RAGE were related to PCOS and treatment. Conclusions. CDD can effectively improve the symptoms of PCOS, and our network pharmacological analysis lays the foundation for future clinical research.
\end{abstract}

\section{Background}

Polycystic ovary syndrome (PCOS) is the most common endocrine disease in women and is characterized by abnormal adrenal and ovarian androgen secretion, ovulatory dysfunction, menstrual irregularity, acne [1], and polycystic ovarian morphological features, and in a significant proportion of patients, insulin resistance, with a prevalence between $5 \%$ and $15 \%[2,3]$. Although for a long period, studies on PCOS have focused on reproductive disorders [4], recent evidence suggests that PCOS is a heterogeneous disorder associated with a large number of severe metabolic implications as well as cardiovascular disease for affected women [5], which brings a heavy burden to the patient's family and to society. However, there is a generally poor understanding of its aetiology [6]. Genome-wide and molecular mechanism studies have identified certain candidate gene targets, although their role in the development of PCOS is still largely unknown; thus, early diagnosis, effective treatment, and the elucidation of underlying mechanisms are necessary $[7,8]$.

In the past few decades, oral contraceptive pills (OCPs) have been widely used in PCOS patients to regulate their menstrual cycles and reduce hyperandrogenism [4]. Clomiphene citrate and letrozole have both been used to induce ovulation in PCOS patients with fertility problems [9-11]. However, reports have been coming in regarding the side effects of OCPs in increasing the risk of venous thromboembolism (VTE) [12, 13]. Moreover, some experts have called attention to the unclear long-term risk-benefit ratio 
$[11,14,15]$. Due to the limitations of current treatments, traditional Chinese medicine (TCM) treatment for PCOS has become an important alternative therapy.

There is no specific Chinese medical term referring to "PCOS" in the ancient books of Chinese medicine. According to the clinical characteristics of PCOS, it is attributed to the category of irregular menstruation, infertility, obesity, etc. For thousands of years, TCM has been used to treat menstrual disorders, infertility, and obesity. There are a number of herbal formulas in treating the above diseases. A study showed that herbal medicine administration significantly relieved some of the symptoms of PCOS [16]. Specifically, the CDD consists of nine herbs: Atractylodes lancea (Thunb.) Dc (Cangzhu), Cyperi Rhizoma (Xiangfu), Arisaematis Rhizoma (Dannanxing), Arum ternatum Thunb (Banxia), Zingiber officinale Roscoe (Shengjiang), Citrus reticulata (Chenpi), Aurantii Fructus (Zhike), Poria cocos (Schw.) Wolf (Fuling), and Licorice (Gancao) and is one of the most common prescriptions for phlegm and dampnesstype PCOS patients [17]. Another study showed that the CDD can significantly improve the pregnancy rate of infertile patients with PCOS, which might be associated with reduced insulin resistance, improved endometrial blood flow, and finally improved endometrial receptivity [18]. Although the CDD has been used clinically for gynaecological diseases for a long time, its mechanism of action is unclear because of its complex composition.

Therefore, it is necessary to clarify the biological basis and molecular nature of the TCM decoction. Network pharmacology has recently been developed as a new strategy and technique for elucidating complex pharmacological problems for new drug discovery [19]. In recent years, the TCM Systems Pharmacology (TCMSP) database and analysis platform has emerged as an ideal information convergence of the absorption, distribution, metabolism, and excretion (ADME) properties, drug-likeness, drug targets, associated diseases, and interaction networks of traditional medicines [20].

TCM decoctions have complex ingredients and multiple targets, and network pharmacology can predict novel compound targets and the potential pathways of action based on existing TCM decoctions and has helped clarify the mechanism of several TCM decoctions so far [21-23].

In this study, we used the network pharmacology approach to explore the potential mechanism of action of CDD in treating PCOS. We first filtered the TCMSP database for active compounds of CDD and identified the targets, followed by mining for disease-related genes and network analysis of those genes (Figure 1).

\section{Methods}

2.1. Chemical Components of Each Herb in CDD. To screen the active ingredients of CDD, we used the TCMSP database (http://tcmspw.com/tcmsp.php) [20]. TCMSP is a unique systems pharmacology platform specifically designed for Chinese medicine that contains information on their ADME characteristics and captures the relationships between disease, targets, ingredients, and drugs [24].
2.2. Pharmacokinetic Prediction of $C D D$. CDD active ingredients were filtered mainly on the basis of oral bioavailability (OB), Caco-2 permeability (Caco-2), and drug-likeness (DL). OB, Caco-2, and DL are the three most key indicators of pharmacology. Specifically, the ingredients contained in the CDD meeting the criteria of $\mathrm{OB} \geq 30 \%$, Caco- $2 \geq 0.4$, and DL $\geq 0.18$ were chosen as candidate ingredients for further analysis.

$\mathrm{OB}$ refers to the percentage and rate of the release and absorption of active ingredients into the systemic blood circulation and is an important pharmacokinetic index of oral drugs [25]. It is also an important index to objectively evaluate the intrinsic quality of oral drugs [26], which is particularly important in the drug administration of most oral Chinese herbal formulas [27]. DL is defined as a complex balance of structural features and various molecular properties, which determine whether the particular molecule is similar to the known drugs [26]. These parameters, such as hydrogen bonding characteristics and hydrophobicity, mainly influence the behaviour of molecules in living organisms, which ultimately affects their transport properties, affinity for proteins, metabolic stability, and many other properties. Caco-2 permeability is widely used as a standard permeability screening assay for oral drug absorption related to drug permeability, which can predict the intestinal absorption of the ingredients and the fraction of the oral dose absorbed in humans [28].

In this study, $\mathrm{OB} \geq 30 \%, \mathrm{DL} \geq 0.18$, and Caco- $2 \geq 0.4$ were regarded as a threshold for filtering possible candidate drugs.

2.3. Potential Targets of the Chemical Components of CDD. We chose the TCMSP database as the main source of component-target data and obtained the target protein names of each herb in CDD. Only the proteins that had interactions with the bioactive components in CDD we had already obtained were selected. Then, we converted the target protein names of the bioactive components of CDD into gene names with the species limited into "Homo sapiens" with the UniProt Knowledgebase (UniProtKB, http:// www.uniprot.org).

2.4. Known Therapeutic Targets for PCOS. We collected PCOS targets from two sources. One is the GeneCards database (https://www.genecards.org/), which is a searchable, integrative database that predicts human genes. The knowledgebase automatically integrates gene-centric data from $\sim 150$ web sources, including genomic, transcriptomic, proteomic, genetic, clinical, and functional information [29]. Another resource was the gene map in the Online Mendelian Inheritance in Man (OMIM) database [30] (https://omim. org/, updated on November 15, 2019), which is a comprehensive, authoritative, and timely knowledgebase of human genes and genetic disorders in the human genome. We searched the OMIM database with the keyword "PCOS."

2.5. Protein-Protein Interactions. To illustrate the possible interaction between PCOS-related targets and the potential 


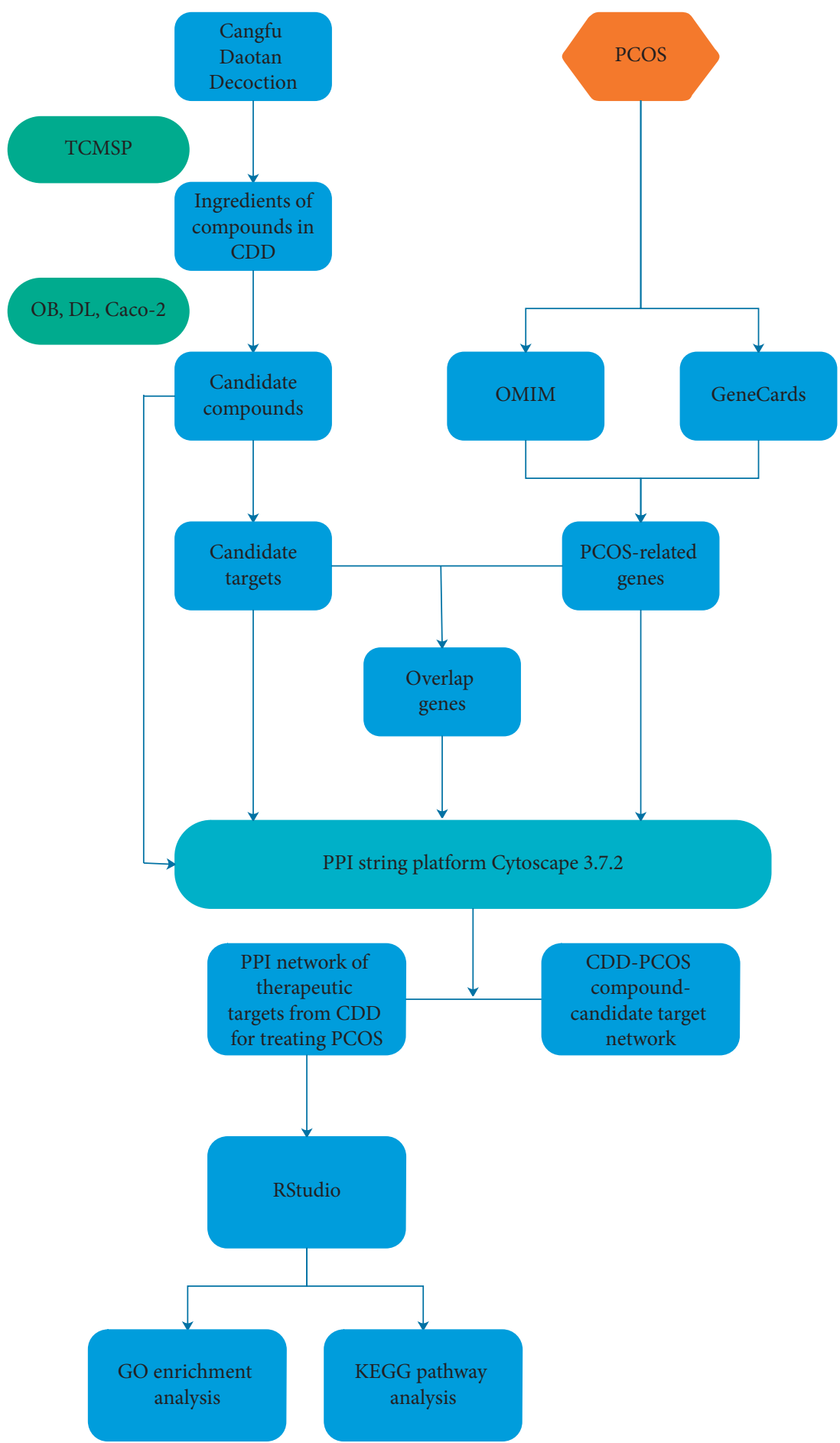

FIGURE 1: A schematic of the network pharmacology-based analysis of CDD for the treatment of PCOS.

targets of CDD, we intersected the potential targets of CDD and PCOS-related drug targets and obtained the intersecting targets with RStudio 3.6.1 (Venn Diagram). The overlapping target proteins of PCOS and CDD were used to construct a protein-protein interaction (PPI) network with multiple protein patterns on the Search Tool for the Retrieval of
Interacting Genes/Proteins (STRING) platform (https:// string-db.org/, version 11.0). We set the organism type to "Homo sapiens" and left the default settings in place for the other parameters. Then, we exported the downloaded "string_interactions.tsv" file and imported it into Cytoscape 3.7.2 to obtain the PPI network and perform network 
analysis. In the network, nodes represent the target proteins, and edges represent the interaction between proteins.

2.6. GO and KEGG Pathway Enrichment Analysis. Gene Ontology (GO) and Kyoto Encyclopedia of Genes and Genomes (KEGG) pathway enrichment analysis are important methods used to describe the characteristics of candidate targets. We selected the standard $p$ value cutoff of 0.05 and the $q$ value of 0.05 and performed the enrichment analysis with RStudio 3.6.1 (Bioconductor, clusterProfiler).

\section{Results}

3.1. Composite Ingredients of CDD. A total of 1051 chemical ingredients of the nine herb medicines in CFDT were retrieved from TCMSP, including 49 ingredients in Atractylodes lancea (Thunb.) Dc, 104 ingredients in Cyperi Rhizoma, 123 ingredients in Arisaematis Rhizoma, 116 ingredients in Arum ternatum Thunb., 265 ingredients in Zingiber officinale Roscoe, 63 ingredients in Citrus reticulata, 17 ingredients in Aurantii Fructus, 34 ingredients in Poria $\operatorname{cocos}(S c h w$.) Wolf, and 280 ingredients in Licorice. 122 ingredients passed the filters of $\mathrm{OB} \geq 30 \%, \mathrm{DL} \geq 0.18$, and Caco- $2 \geq 0.4$. The pharmacokinetic properties of the compounds are shown in Table 1.

3.2. Target Gene Prediction of CDD. A total of 1433 potential targets from the 111 ingredients were retrieved from the TCMSP database (Figure 2). After eliminating the overlapping proteins, 118 related proteins were obtained and converted into gene names with the species limited into "Homo sapiens" based on the UniProtKB.

3.3. PCOS-Related Target Network. Research has shown that PCOS is a genetic predisposing, complex polygenic, multifactorial disorder $[7,31]$. In this study, we obtained 736 targets related to PCOS through the GeneCards database (https://www.genecards.org/) and the OMIM database (https://omim.org/).

3.4. PPI Network Analysis. Among the above 736 PCOSrelated targets, CDD shared 44 common targets with PCOS (Figure 3). The 44 putative therapeutic targets were imported into the STRING database to establish the putative therapeutic target PPI network. The "string_interaction.tsv" file was then imported into Cytoscape 3.7.2 to perform network analysis. The network had 44 nodes, which interacted with 361 edges. The average node degree is 16.4 , and the average local clustering coefficient is 0.698 . From yellow to green, the degrees of freedom increase, and the thicker edges suggest stronger interactions (Figure 4). Our results indicated that the top mutual target proteins have various beneficial functions for treating PCOS at the molecular level.

In our network, the degree of the greenness of the nodes is high, and the numbers of edges of the nodes are 37 for AKT1; 32 for IL-6; 29 for VEGFA; 28 for CCND1, STAT3, MAPK1, and MAPK8; 27 for ESR1; 26 for FN1; 25 for
MMP9, CXCL8, and MAPK14; 23 for AR; and 22 for PPARG and HIF1A. These results demonstrate that these targets are closely related to others in the network and consequently may play key roles in PCOS.

AKTs have been confirmed to play an important role in granulosa-lutein cell (GC) proliferation, and there is reciprocal feedback between AKT and androgen. This study showed that the high expression of AKT1 and AKT2 may cause GC dysfunction in PCOS patients through a possible relation with androgen [32]. Elevated serum levels of IL-6 in PCOS patients reflect low-grade chronic inflammation, which has been attributed to insulin resistance in PCOS [33]. In a PCOS rat model, increased expression of IL- 6 and IL-11 was found to be associated with the AKT/STAT3 pathway. Increased levels of IL- 6 and IL-11 stimulated adipocytes from adipose tissue of the PCOS rat model, which may activate AKT/STAT3 signalling and promote cell proliferation. Vascular endothelial growth factor (VEGF) plays an important role in the pathogenesis of many diseases. PCOS was also considered to be associated with high expression levels of VEGF. It was found that VEGF was a pivotal mediator of other factors to control ovary angiogenesis in women who underwent assisted reproductive technology (ART) procedures [34].

The MAPK signalling pathway is one of the most important signal transduction pathways in organisms and mediates physiological and pathological processes, such as the growth, development, and differentiation of organisms. Studies have shown that the MAPK signalling pathway plays an important role in the proliferation of ovarian granulosa cells $[35,36]$.

Research confirmed that PPARG rs709154 and ESR1 rs1999805 are significantly associated with PCOS risk in a Chinese population [37]. MMP9 may also be involved in the pathogenesis of PCOS [38]. HIF1A can induce glycolysis gene expression and promote anaerobic metabolism [39]. HIF-1a signalling was confirmed to be inhibited in a rat model with PCOS by increasing PHD activity [40].

\subsection{Putative Therapeutic Compound-Putative Therapeutic} Target Network Construction. The Merge function of Cytoscape 3.7.2 software was used to combine the DrugMol-Gene target network and the PCOS-Drug-Gene interaction network. The results showed that 111 compounds all together play a role in treating PCOS in the composite (Figure 5). The targets of 111 putative therapeutic targets in the treatment of PCOS include AKT1, IL-6, VEGFA, CCND1, STAT3, MAPK1, MAPK8, ESR1, FN1, MMP9, CXCL8, MAPK14, AR, PPARG, and HIF1A.

3.6. GO Functional Enrichment Analysis. To further illuminate the biological effects involved in the treatment of PCOS with CDD, we performed GO analysis of the 44 PCOS-related putative potential therapeutic target genes. GO annotation and enrichment of the genes encoding CDD were conducted from three aspects: molecular function (MF), biological process (BP), and cellular composition 
TABLE 1: A list of the active compounds in CDD.

\begin{tabular}{|c|c|c|c|c|}
\hline Mol ID & Molecule name & 0B (\%) & Caco-2 & $\mathrm{DL}$ \\
\hline MOL001484 & Inermine & 75.18 & 0.89 & 0.54 \\
\hline MOL001792 & DFV & 32.76 & 0.51 & 0.51 \\
\hline MOL000211 & Mairin & 55.38 & 0.73 & 0.78 \\
\hline MOL002311 & Glycyrol & 90.78 & 0.71 & 0.67 \\
\hline MOL000239 & Jaranol & 50.83 & 0.61 & 0.29 \\
\hline MOL002565 & Medicarpin & 49.22 & 1 & 0.34 \\
\hline MOL000359 & Sitosterol & 36.91 & 1.32 & 0.75 \\
\hline MOL003656 & Lupiwighteone & 51.64 & 0.68 & 0.37 \\
\hline MOL003896 & 7-Methoxy-2-methyl isoflavone & 42.56 & 1.16 & 0.2 \\
\hline MOL000392 & Formononetin & 69.67 & 0.78 & 0.21 \\
\hline MOL000417 & Calycosin & 47.75 & 0.52 & 0.25 \\
\hline MOL004805 & $\begin{array}{c}\text { (2S)-2-[4-Hydroxy-3-(3-methylbut-2-enyl)phenyl]-8,8-dimethyl-2,3-dihydropyrano[2,3-f] } \\
\text { chromen-4-one }\end{array}$ & 31.79 & 1 & 0.72 \\
\hline MOL004806 & Euchrenone & 30.29 & 1.09 & 0.57 \\
\hline MOL004808 & Glyasperin B & 65.22 & 0.47 & 0.44 \\
\hline MOL004810 & Glyasperin F & 75.84 & 0.43 & 0.54 \\
\hline MOL004811 & Glyasperin C & 45.56 & 0.71 & 0.4 \\
\hline MOL004814 & Isotrifoliol & 31.94 & 0.53 & 0.42 \\
\hline MOL004815 & (E)-1-(2,4-Dihydroxyphenyl)-3-(2,2-dimethylchromen-6-yl)prop-2-en-l-one & 39.62 & 0.66 & 0.35 \\
\hline MOL004820 & Kanzonol W & 50.48 & 0.63 & 0.52 \\
\hline MOL004827 & Semilicoisoflavone B & 48.78 & 0.45 & 0.55 \\
\hline MOL004828 & Glepidotin A & 44.72 & 0.79 & 0.35 \\
\hline MOL004829 & Glepidotin B & 64.46 & 0.46 & 0.34 \\
\hline MOL004833 & Phaseolinisoflavan & 32.01 & 1.01 & 0.45 \\
\hline MOL004835 & Glypallichalcone & 61.6 & 0.76 & 0.19 \\
\hline MOL004838 & 8-(6-Hydroxy-2-benzofuranyl)-2,2-dimethyl-5-chromenol & 58.44 & 1 & 0.38 \\
\hline MOL004841 & Licochalcone B & 76.76 & 0.47 & 0.19 \\
\hline MOL004848 & Licochalcone G & 49.25 & 0.64 & 0.32 \\
\hline MOL004849 & 3-(2,4-Dihydroxyphenyl)-8-(1,1-dimethylprop-2-enyl)-7-hydroxy-5-methoxy-coumarin & 59.62 & 0.4 & 0.43 \\
\hline MOL004855 & Licoricone & 63.58 & 0.53 & 0.47 \\
\hline MOL004856 & Gancaconin A & 51.08 & 0.8 & 0.4 \\
\hline MOL004857 & Gancaconin B & 48.79 & 0.58 & 0.45 \\
\hline MOL004863 & 3-(3,4-Dihydroxyphenyl)-5,7-dihydroxy-8-(3-methylbut-2-enyl)chromone & 66.37 & 0.52 & 0.41 \\
\hline MOL004864 & 5,7-Dihydroxy-3-(4-methoxyphenyl)-8-(3-methylbut-2-enyl)chromone & 30.49 & 0.9 & 0.41 \\
\hline MOL004866 & 2-(3,4-Dihydroxyphenyl)-5,7-dihydroxy-6-(3-methylbut-2-enyl)chromone & 44.15 & 0.48 & 0.41 \\
\hline MOL004879 & Glycyrin & 52.61 & 0.59 & 0.47 \\
\hline MOL004882 & Licocoumarone & 33.21 & 0.84 & 0.36 \\
\hline MOL004884 & Licoisoflavone B & 38.93 & 0.46 & 0.55 \\
\hline MOL004891 & Shinpterocarpin & 80.3 & 1.1 & 0.73 \\
\hline MOL004898 & (E)-3-[3,4-Dihydroxy-5-(3-methylbut-2-enyl)phenyl]-1-(2,4-dihydroxyphenyl)prop-2-en-1-one & 46.27 & 0.41 & 0.31 \\
\hline MOL004910 & Glabridin & 53.25 & 0.97 & 0.47 \\
\hline MOL004911 & Glabridin & 52.9 & 0.97 & 0.31 \\
\hline MOL004912 & Glabrone & 52.51 & 0.59 & 0.5 \\
\hline MOL004913 & 1,3-Dihydroxy-9-methoxy-6-benzofurano[3,2-c]chromone & 48.14 & 0.48 & 0.43 \\
\hline MOL004915 & Eurycarpin A & 43.28 & 0.43 & 0.37 \\
\hline MOL004935 & Sigmoidin B & 34.88 & 0.42 & 0.41 \\
\hline MOL004941 & (2R)-7-Hydroxy-2-(4-hydroxyphenyl)chroman-4-one & 71.12 & 0.41 & 0.18 \\
\hline MOL004945 & (2S)-7-Hydroxy-2-(4-hydroxyphenyl)-8-(3-methylbut-2-enyl)chromen-4-one & 36.57 & 0.72 & 0.32 \\
\hline MOL004948 & Isoglycyrol & 44.7 & 0.91 & 0.84 \\
\hline MOL004949 & Isolicoflavonol & 45.17 & 0.54 & 0.42 \\
\hline MOL004957 & HMO & 38.37 & 0.79 & 0.21 \\
\hline MOL004959 & 1-Methoxyphaseollidin & 69.98 & 1.01 & 0.64 \\
\hline MOL004966 & $3^{\prime}$-Hydroxy- $4^{\prime}-0$-methylglabridin & 43.71 & 1 & 0.57 \\
\hline MOL000497 & Licochalcone A & 40.79 & 0.82 & 0.29 \\
\hline MOL004974 & $3^{\prime}$-Methylglabridin & 46.16 & 0.94 & 0.57 \\
\hline MOL004978 & 2-[(3R)-8,8-Dimethyl-3,4-dihydro-2H-pyrano[6,5-f]chromen-3-yl]-5-methoxyphenol & 36.21 & 1.12 & 0.52 \\
\hline MOL004980 & Inflacoumarin A & 39.71 & 0.73 & 0.33 \\
\hline MOL004985 & Icso-5-enoic acid & 30.7 & 1.22 & 0.2 \\
\hline MOL004988 & Kanzonol F & 32.47 & 1.18 & 0.89 \\
\hline MOL004991 & 7-Acetoxy-2-methylisoflavone & 38.92 & 0.74 & 0.26 \\
\hline
\end{tabular}


TABle 1: Continued.

\begin{tabular}{|c|c|c|c|c|}
\hline Mol ID & Molecule name & OB (\%) & Caco-2 & $\mathrm{DL}$ \\
\hline MOL004993 & 8-Prenylated eriodictyol & 53.79 & 0.43 & 0.4 \\
\hline MOL004996 & Gadelaidic acid & 30.7 & 1.2 & 0.2 \\
\hline MOL000500 & Vestitol & 74.66 & 0.86 & 0.21 \\
\hline MOL005000 & Gancaonin G & 60.44 & 0.78 & 0.39 \\
\hline MOL005001 & Gancaonin $\mathrm{H}$ & 50.1 & 0.6 & 0.78 \\
\hline MOL005003 & Licoagrocarpin & 58.81 & 1.23 & 0.58 \\
\hline MOL005007 & Glyasperin M & 72.67 & 0.49 & 0.59 \\
\hline MOL005012 & Licoagroiosoflavone & 57.28 & 0.71 & 0.49 \\
\hline MOL005016 & Odoratin & 49.95 & 0.42 & 0.3 \\
\hline MOL005017 & Phaseol & 78.77 & 0.76 & 0.58 \\
\hline MOL005018 & Xambioona & 54.85 & 1.09 & 0.87 \\
\hline MOL005020 & Dehydroglyasperin C & 53.82 & 0.68 & 0.37 \\
\hline MOL000173 & Wogonin & 30.68 & 0.79 & 0.23 \\
\hline MOL000184 & NSC63551 & 30.25 & 1.42 & 0.76 \\
\hline MOL000186 & Stigmasterol 3-0-beta-D-glucopyranoside_qt & 43.83 & 1.31 & 0.76 \\
\hline MOL000188 & $3 \beta$-Acetoxyatractylone & 40.57 & 1.22 & 0.22 \\
\hline MOL000085 & Beta-daucosterol_qt & 36.91 & 1.3 & 0.75 \\
\hline MOL000088 & Beta-sitosterol 3-0-glucoside_qt & 36.91 & 1.3 & 0.75 \\
\hline MOL000092 & Daucosterin_qt & 36.91 & 1.42 & 0.76 \\
\hline MOL000094 & Daucosterol_qt & 36.91 & 1.3 & 0.76 \\
\hline MOL003542 & 8-Isopentenyl-kaempferol & 38.04 & 0.53 & 0.39 \\
\hline MOL000358 & Beta-sitosterol & 36.91 & 1.32 & 0.75 \\
\hline MOL004027 & 1,4-Epoxy-16-hydroxypheneicos-1,3,12,14,18-pentaene & 45.1 & 1.28 & 0.24 \\
\hline MOL004053 & Isodalbergin & 35.45 & 0.8 & 0.2 \\
\hline MOL004058 & Khell & 33.19 & 1.12 & 0.19 \\
\hline MOL004068 & Rosenonolactone & 79.84 & 0.72 & 0.37 \\
\hline MOL004071 & Hyndarin & 73.94 & 1 & 0.64 \\
\hline MOL004074 & Stigmasterol glucoside_qt & 43.83 & 1.31 & 0.76 \\
\hline MOL004077 & Sugeonyl acetate & 45.08 & 0.72 & 0.2 \\
\hline MOL000449 & Stigmasterol & 43.83 & 1.44 & 0.76 \\
\hline MOL013146 & $8,11,14$-Docosatrienoic acid, methyl ester & 43.23 & 1.53 & 0.3 \\
\hline MOL001510 & 24-Epicampesterol & 37.58 & 1.43 & 0.71 \\
\hline MOL000953 & CLR & 37.87 & 1.43 & 0.68 \\
\hline MOL001755 & 24-Ethylcholest-4-en-3-one & 36.08 & 1.46 & 0.76 \\
\hline MOL002670 & Cavidine & 35.64 & 1.08 & 0.81 \\
\hline MOL002714 & Baicalein & 33.52 & 0.63 & 0.21 \\
\hline MOL005030 & Gondoic acid & 30.7 & 1.2 & 0.2 \\
\hline MOL000519 & Coniferin & 31.11 & 0.42 & 0.32 \\
\hline MOL006936 & 10,13-Eicosadienoic & 39.99 & 1.22 & 0.2 \\
\hline MOL006957 & (3S,6S)-3-(Benzyl)-6-(4-hydroxybenzyl)piperazine-2,5-quinone & 46.89 & 0.41 & 0.27 \\
\hline MOL003578 & Cycloartenol & 38.69 & 1.53 & 0.78 \\
\hline MOL006129 & 6-Methylgingediacetate2 & 48.73 & 0.55 & 0.32 \\
\hline MOL001771 & Poriferast-5-en-3beta-ol & 36.91 & 1.45 & 0.75 \\
\hline MOL008698 & Dihydrocapsaicin & 47.07 & 0.98 & 0.19 \\
\hline MOL005815 & Citromitin & 86.9 & 0.88 & 0.51 \\
\hline MOL005828 & Nobiletin & 61.67 & 1.05 & 0.52 \\
\hline MOL000275 & Trametenolic acid & 38.71 & 0.52 & 0.8 \\
\hline MOL000282 & Ergosta-7,22E-dien-3beta-ol & 43.51 & 1.32 & 0.72 \\
\hline MOL000283 & Ergosterol peroxide & 40.36 & 0.84 & 0.81 \\
\hline MOL000287 & 3beta-Hydroxy-24-methylene-8-lanostene-21-oic acid & 38.7 & 0.61 & 0.81 \\
\hline MOL000296 & Hederagenin & 36.91 & 1.32 & 0.75 \\
\hline
\end{tabular}

(CC). The most enriched terms in GO analysis are shown in Figures 6-8.

In detail, the top 18 terms in the GO BP category were mainly enriched in the regulation of developmental growth, reproductive system development, response to steroid hormone, epithelial cell proliferation, gland development, regulation of epithelial cell proliferation, transcription initiation from RNA polymerase II promoter, female pregnancy, multi-multicellular organism process, DNAtemplated transcription, initiation, muscle cell proliferation, ossification, regulation of DNA-binding transcription factor activity, response to radiation, ameboidal-type cell 


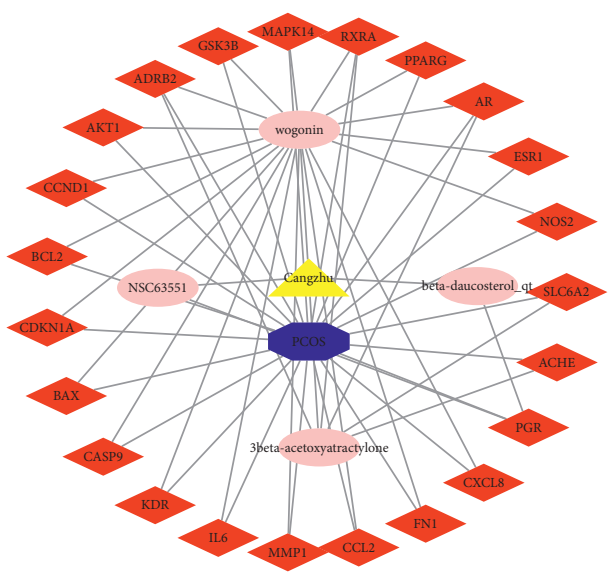

(a)

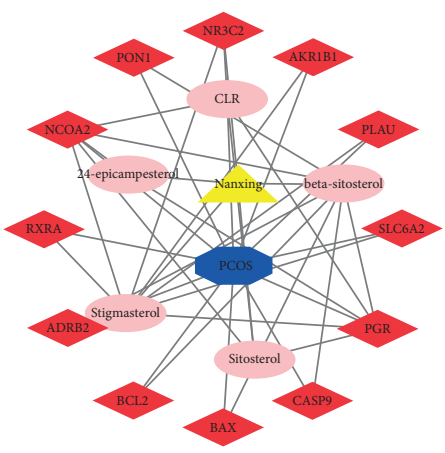

(c)

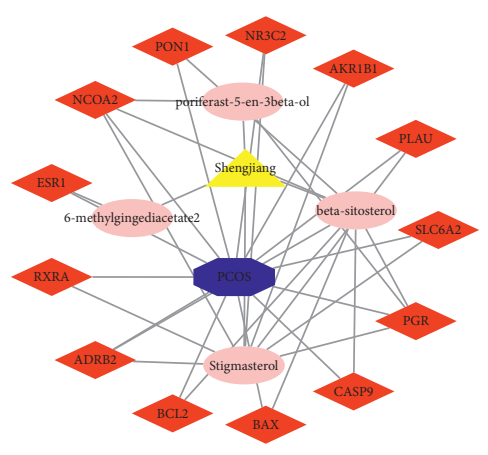

(e)

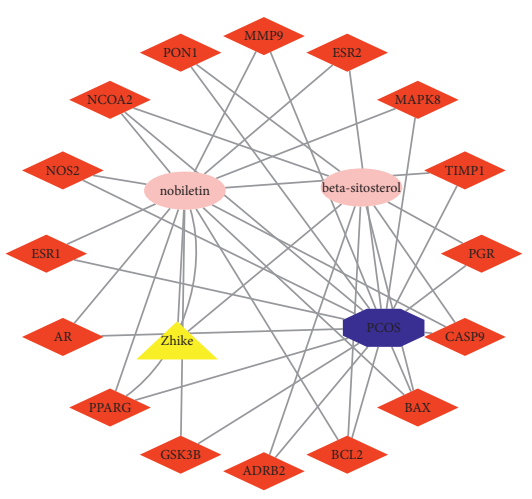

(g)

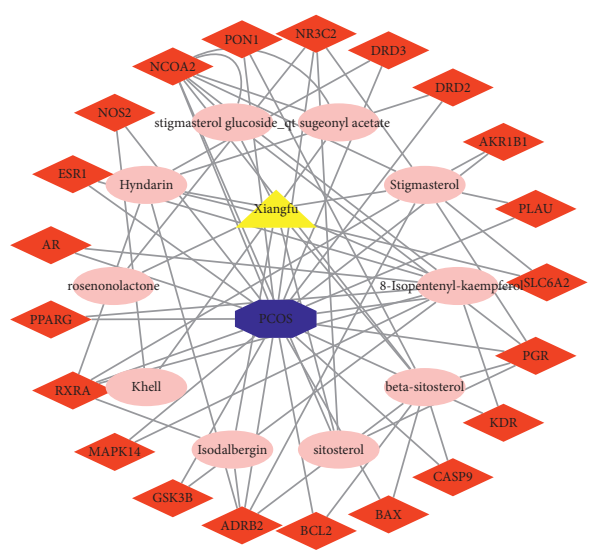

(b)

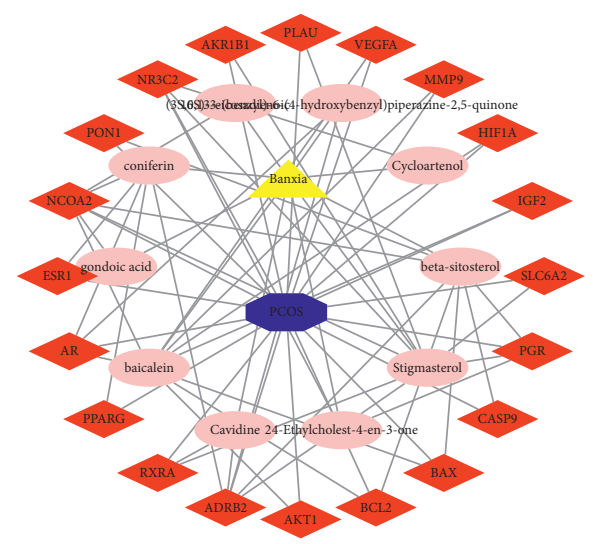

(d)

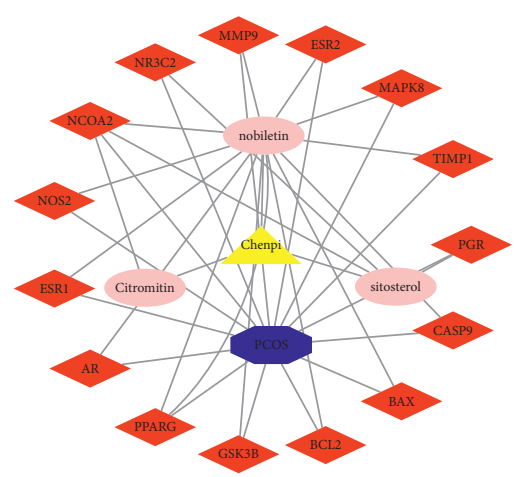

(f)

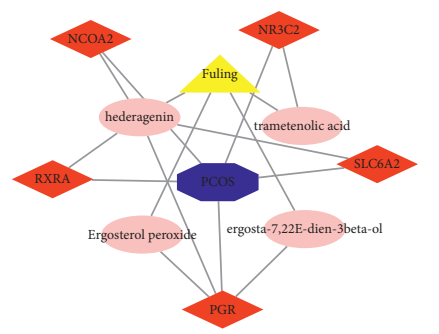

(h)

Figure 2: Continued. 


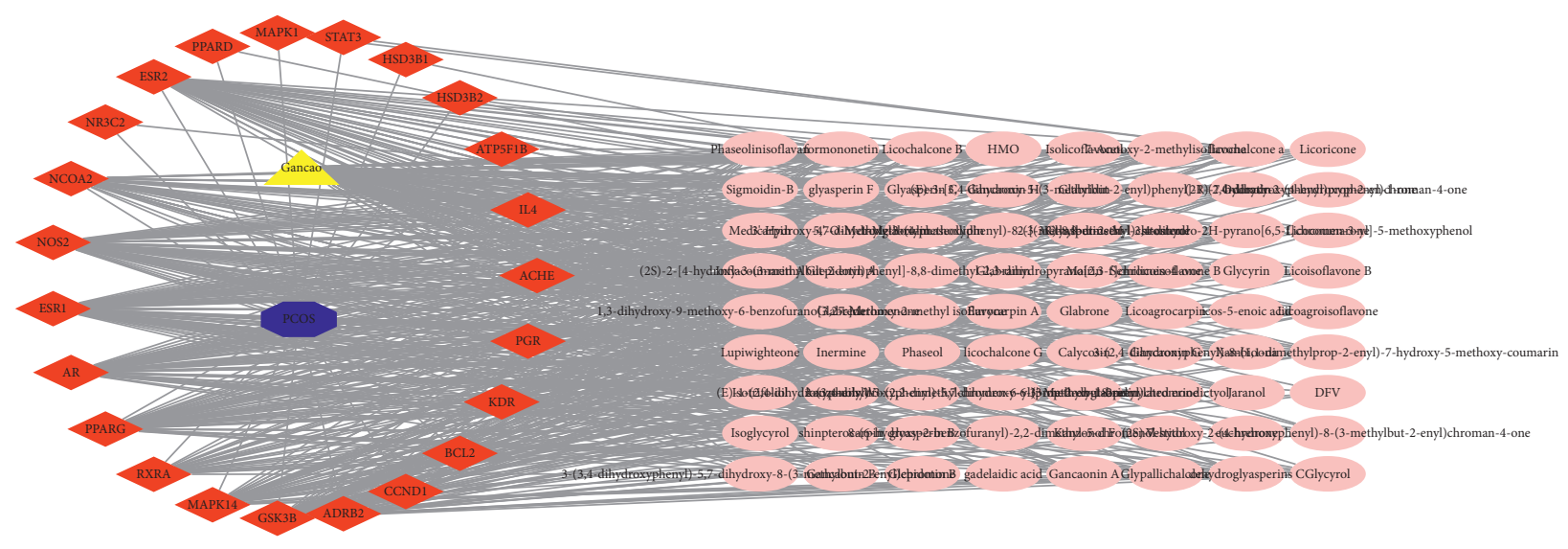

(i)

FIgURE 2: The potential targets from the ingredients of the herbs in CDD in the treatment of PCOS. The yellow triangle, red diamond, blue octagon, and the light pink ellipse nodes represent the herbs, targets, disease, and the molecules, respectively.

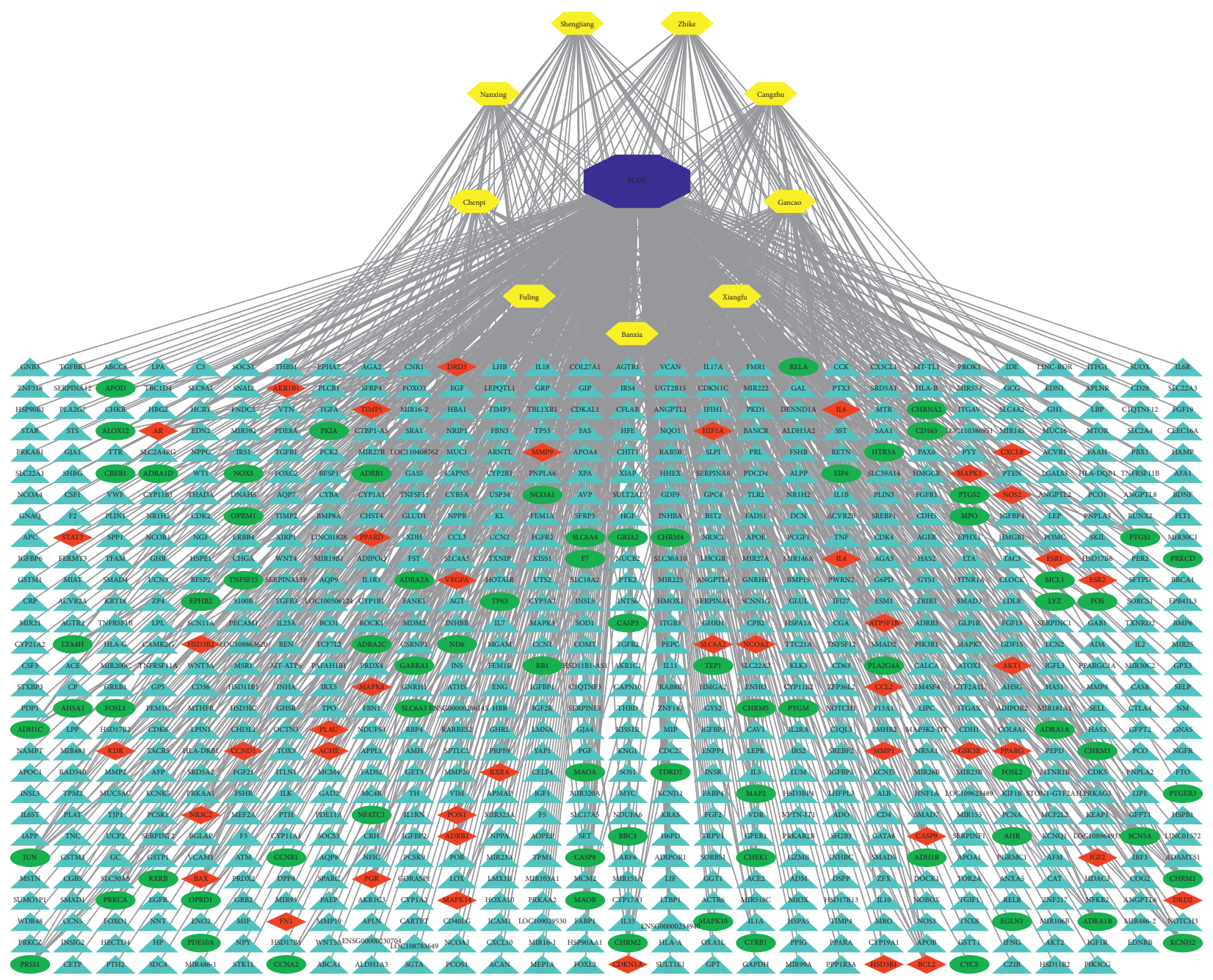

Figure 3: The CDD-PCOS-related drug targets. The blue octagon, yellow hexagon, green ellipse, mint green triangle, and red diamond represent disease, herbs, PCOS-related targets, CDD-related targets, and the common targets between CDD and PCOS, respectively. 


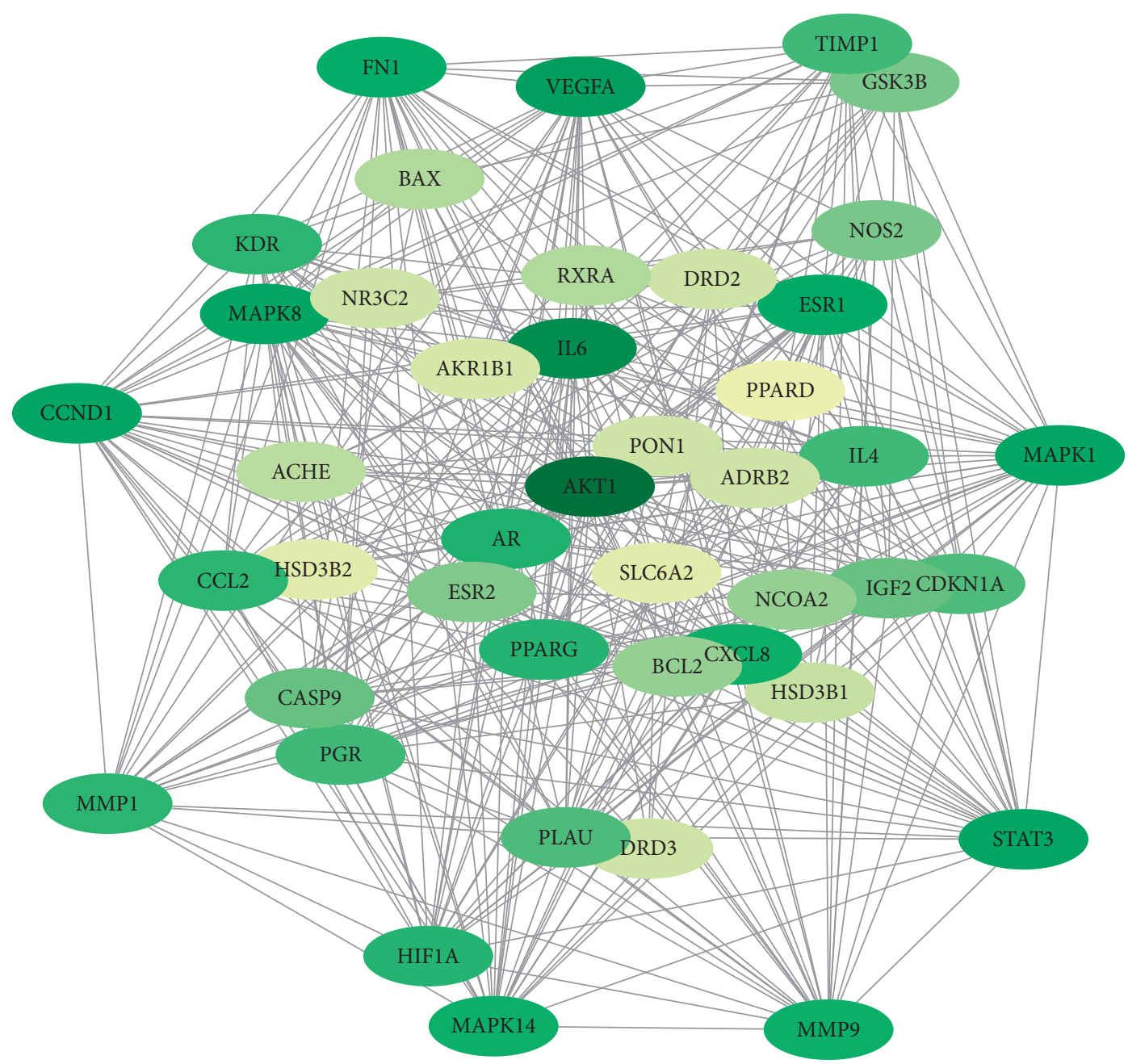

FIGURE 4: PPI network of targets for CDD in treating PCOS (from yellow to green, the degrees of freedom increase, and the thicker edges suggest stronger interactions).

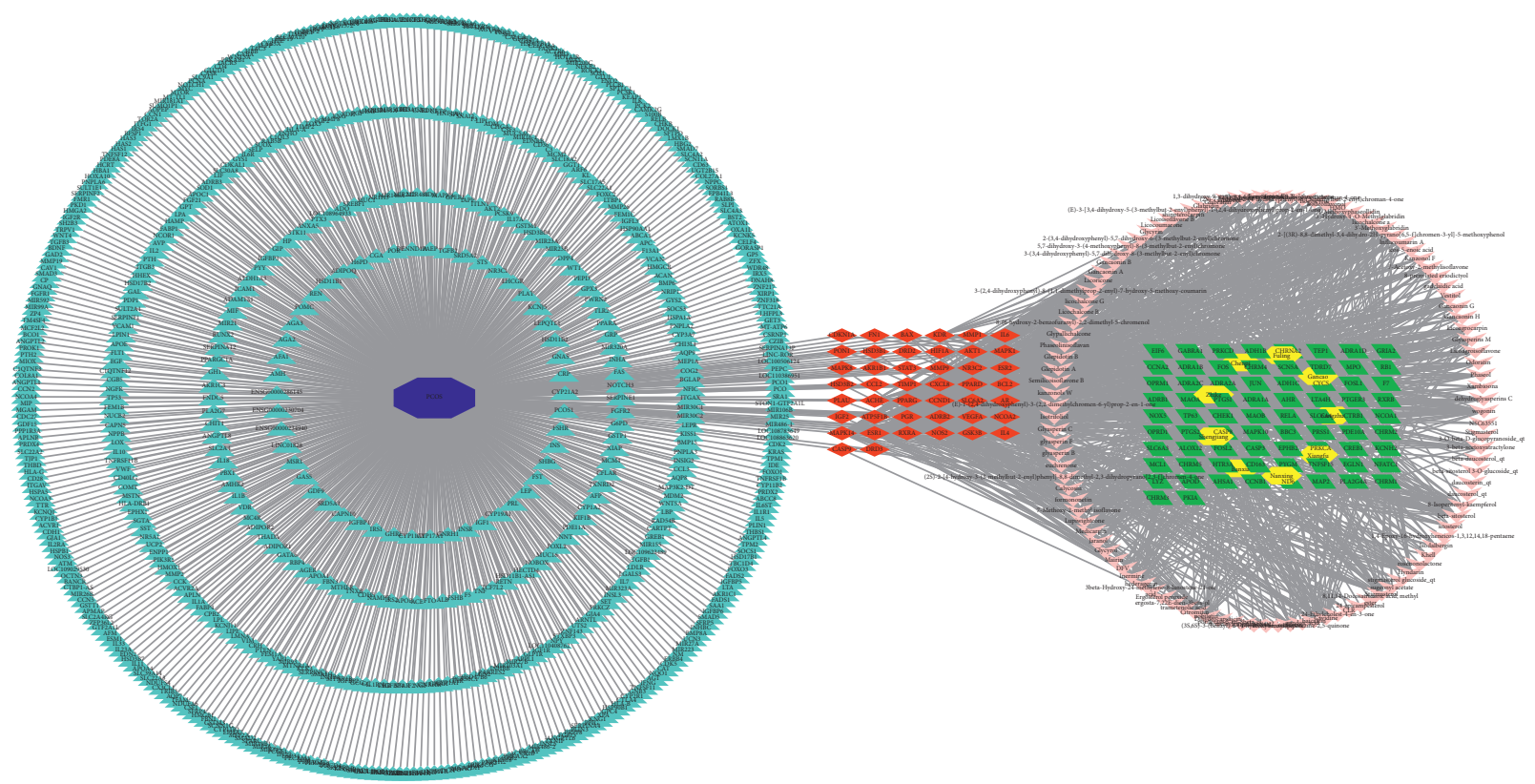

FIGURE 5: Herb-compound target-PCOS target network of CDD. The blue octagon, mint green triangle, yellow hexagon, green parallelogram, pink red V, and red diamond represent disease, PCOS-related targets, herbs, CDD-related targets, molecules, and the common targets, respectively. 


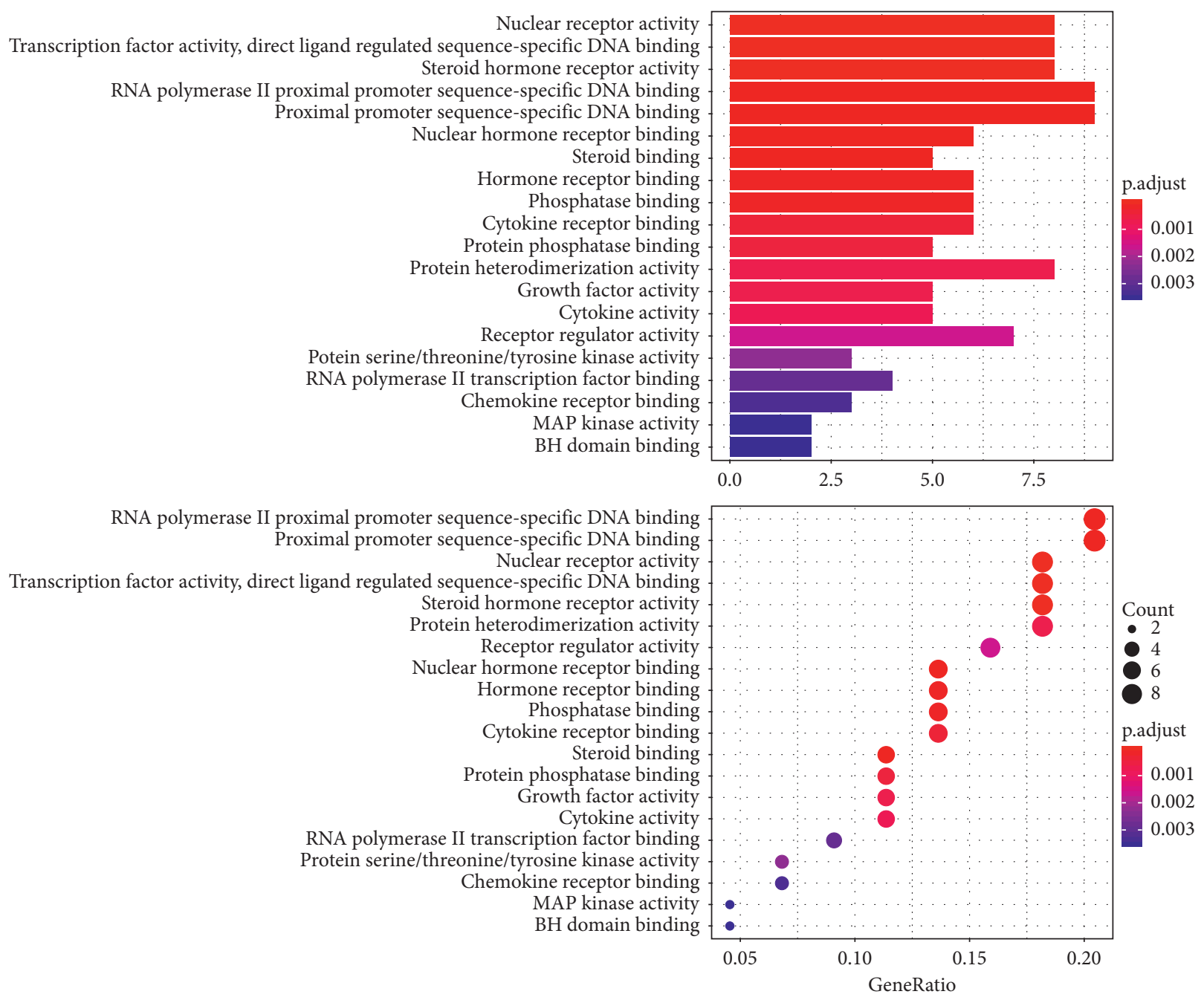

FIGURE 6: GO MF enrichment analysis of therapeutic targets of CDD in treating PCOS.

migration, autophagy, and process utilizing autophagic mechanism (Figure 6).

The top 19 terms in the GO CC category were mainly enriched in nuclear chromatin, secretory granule lumen, cytoplasmic vesicle lumen, vesicle lumen, extracellular matrix, nuclear chromosome part, chromatin, glutamatergic synapse, platelet alpha granule lumen, platelet alpha granule, RNA polymerase II transcription factor complex, nuclear transcription factor complex, transcription factor complex, postsynaptic density, receptor complex, asymmetric synapse, neuron to neuron synapse, postsynaptic specialization, and basement membrane (Figure 7).

The top 19 terms in the GO MF category were mainly enriched in RNA polymerase II proximal promoter sequence-specific DNA binding, proximal promoter sequence-specific DNA binding, nuclear receptor activity, transcription factor activity, direct ligand regulated sequence-specific DNA binding, steroid hormone receptor activity, protein heterodimerization activity, receptor regulator activity, nuclear hormone receptor binding, hormone receptor binding, phosphatase binding, cytokine receptor binding, receptor ligand activity, chromatin binding, steroid binding, protein phosphatase binding, growth factor activity, cytokine activity, protein serine/threonine kinase activity, DNA-binding transcription activator activity, RNA polymerase II-specific, transcription coregulator activity, and RNA polymerase II transcription factor binding (Figure 8).

3.7. Pathway Analysis. To clarify the biological actions of these targets, we performed KEGG analysis and pathway enrichment analysis with the RStudio (Bioconductor) database. We listed the genes of major PCOS putative therapeutic targets and imported them into RStudio to generate relevant pathways that might have an important influence on the biological process of CDD in treating PCOS. Pathways with an adjusted $p$ value $<0.05$ were considered significant.

A total of 123 signalling pathways were significantly enriched through pathway enrichment analysis. In the histogram and bubble diagram (Figure 9), the colour, size, and length of the nodes and bars were determined according to the numbers and $p$ values of the related genes. The colour 

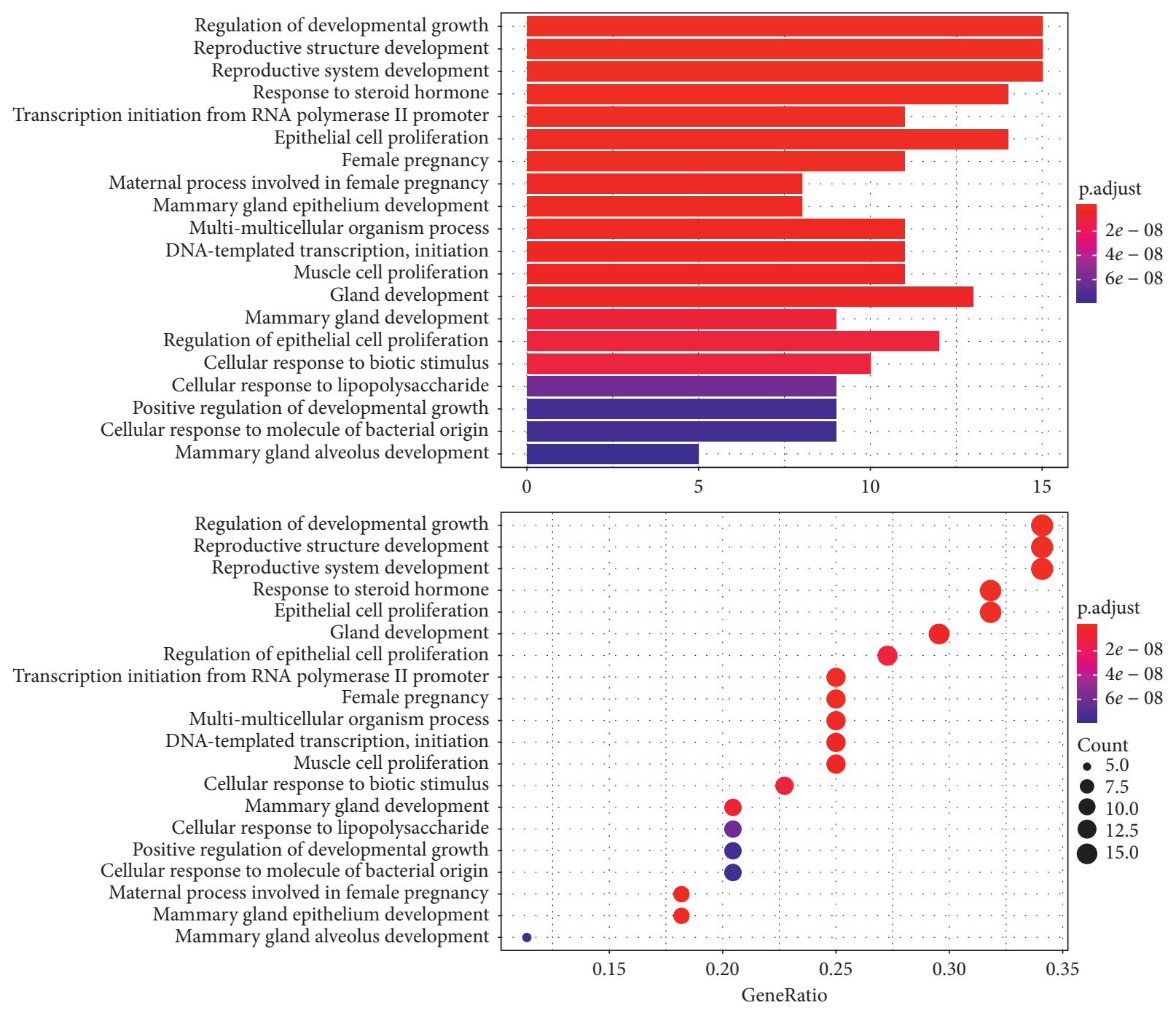

FIgURe 7: GO BP enrichment analysis of therapeutic targets of CDD in treating PCOS.

from blue to red reflects the adjusted $p$ values from large to small, and the node size and bar length indicate the number of related genes. The histogram and bubble chart were separately arranged according to the $p$ value and the number of related genes. According to the $p$ value, the AGE-RAGE signalling pathway in diabetic complications, Kaposi sarcoma-associated herpesvirus infection, proteoglycans in cancer, endocrine resistance, hepatitis B, the IL-17 signalling pathway, prostate cancer, the prolactin signalling pathway, human cytomegalovirus infection, pancreatic cancer, the HIF-1 signalling pathway, and EGFR tyrosine kinase inhibitor resistance were within the top 12 terms. According to the number of related genes, Kaposi sarcoma-associated herpesvirus infection, proteoglycans in cancer, the PI3K-Akt signalling pathway, the AGE-RAGE signalling pathway in diabetic complications, human cytomegalovirus infection, hepatitis $\mathrm{B}$, endocrine resistance, the IL-17 signalling pathway, prostate cancer, the HIF-1 signalling pathway, Epstein-Barr virus infection, and the prolactin signalling pathway were in the top 12 terms. The overlapping terms of the above lists included the AGE-RAGE signalling pathway in diabetic complications, endocrine resistance, the IL-17 signalling pathway, the prolactin signalling pathway, and the HIF-1 signalling pathway.

AGE-RAGE signalling may activate multiple intracellular signalling pathways involving protein kinase $\mathrm{C}$ and MAPKs and may influence NF- $\kappa \mathrm{B}$ activity. Research has shown an association between PCOS and chronic inflammatory factors, proinflammatory cytokines such as interleukin-1, interleukin-6, interleukin-17 [41-43], and tumour necrosis- $\alpha$, and a variety of inflammationrelated genes, including VEGF, tissue factor, and RAGE $[44,45]$.

Prolactin (PRL) is involved in a variety of biological functions, including osmotic regulation, reproduction, lactation, endocrinology and metabolism, growth and development, and immunomodulation. It is a polypeptide hormone secreted by the pituitary gland that regulates the female reproductive and endocrine system by activating pathways, including the MAPK and PI3K pathways [46]. Insulin resistance is a common type of endocrine resistance and is related to PCOS by deregulating the IRS-PI3K-Akt 


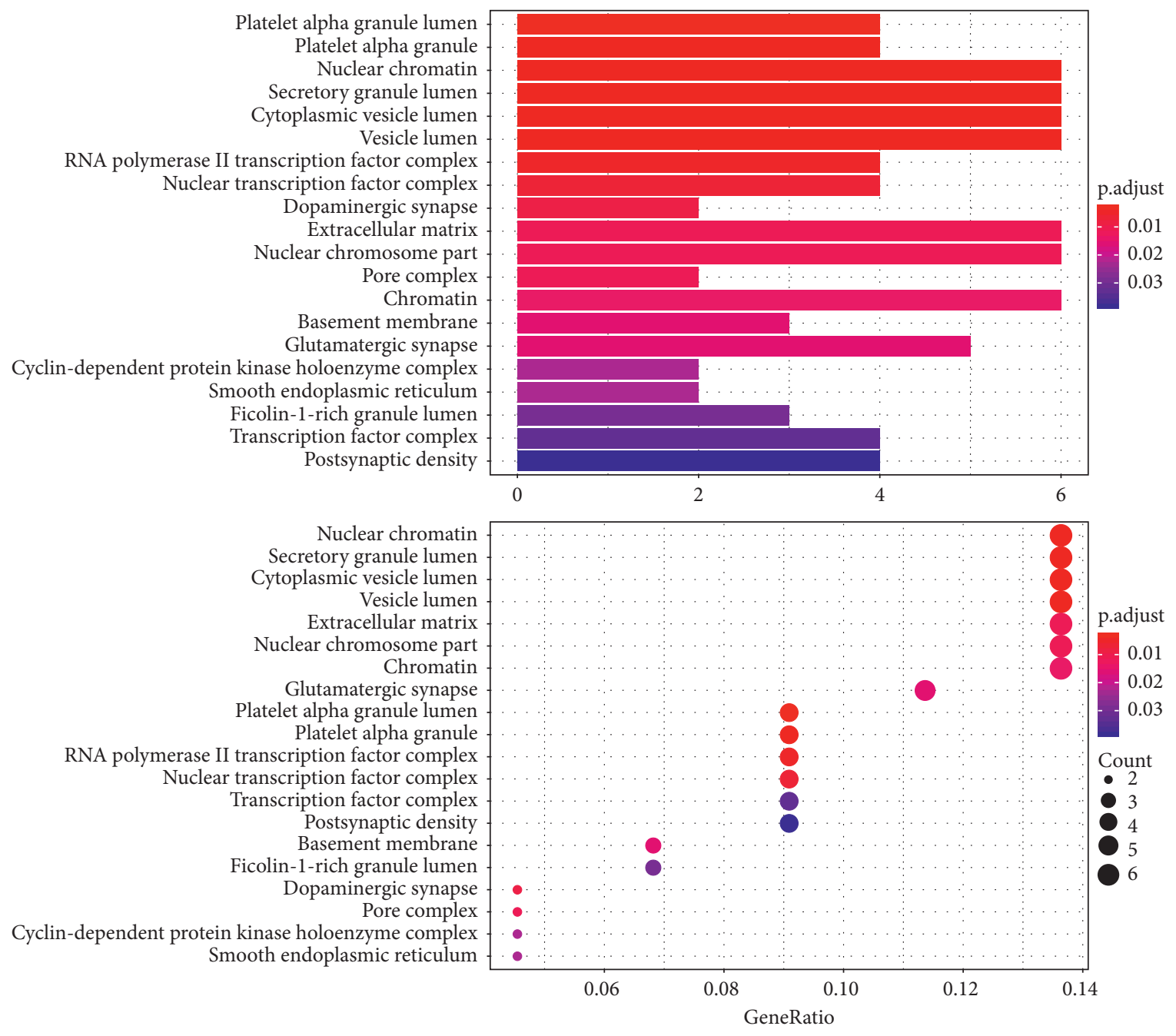

FIGURE 8: GO CC enrichment analysis of therapeutic targets of CDD in treating PCOS.

signalling axis that integrates aberrant inflammatory responses [47].

A previous study also revealed that hypoxia-inducible factor-1a (HIF-1a) mediated endothelin-2 (ET-2) signalling and plays an important role in ovulation in rats and indicated that HIF-1a signalling is inhibited in a PCOS rat model by increasing PHD activity [40].

\section{Discussion}

TCM plays a crucial role in the pharmaceutical industry, alternative medicine, and many other fields [48-50]. However, due to the complex chemical composition and unclear pharmacological mechanism of action of TCMs, it faces great obstacles in pharmacological research, quality control and supervision, modernization, and internationalization [51].

TCM gives priority to the concept of holism in diagnosis and pays more attention to the overall changes in function. The treatment is based on syndrome differentiation, emphasizing the recovery of overall function, which is similar to the network concept of network pharmacology [52].
Traditional research methods on TCM mainly rely on pharmacochemical and pharmacological experiments, which have many problems, such as low data flux, low precision, long cycles, and high costs. The construction and development of a network pharmacology analysis platform, TCM database, and target prediction technology has brought new ideas and strategies for the study on the basis and mechanism of the pharmacodynamic substances of TCM to clarify characteristics such as the good curative effects, high safety profile, and multicomponents and multitargets of TCM [53]. These developments also lead to a new direction for the modernization and internationalization of TCM [54].

The combination of bioinformatics, computer technology, and experiments in the study of the pharmacodynamic components and potential targets of TCM broke through the deficiency of traditional studies is more rapid, flexible, and accurate and has been widely used in research on the network pharmacology of TCM [55].

TCM has a long history in treating menstrual diseases and infertility related to PCOS and has achieved good effects 


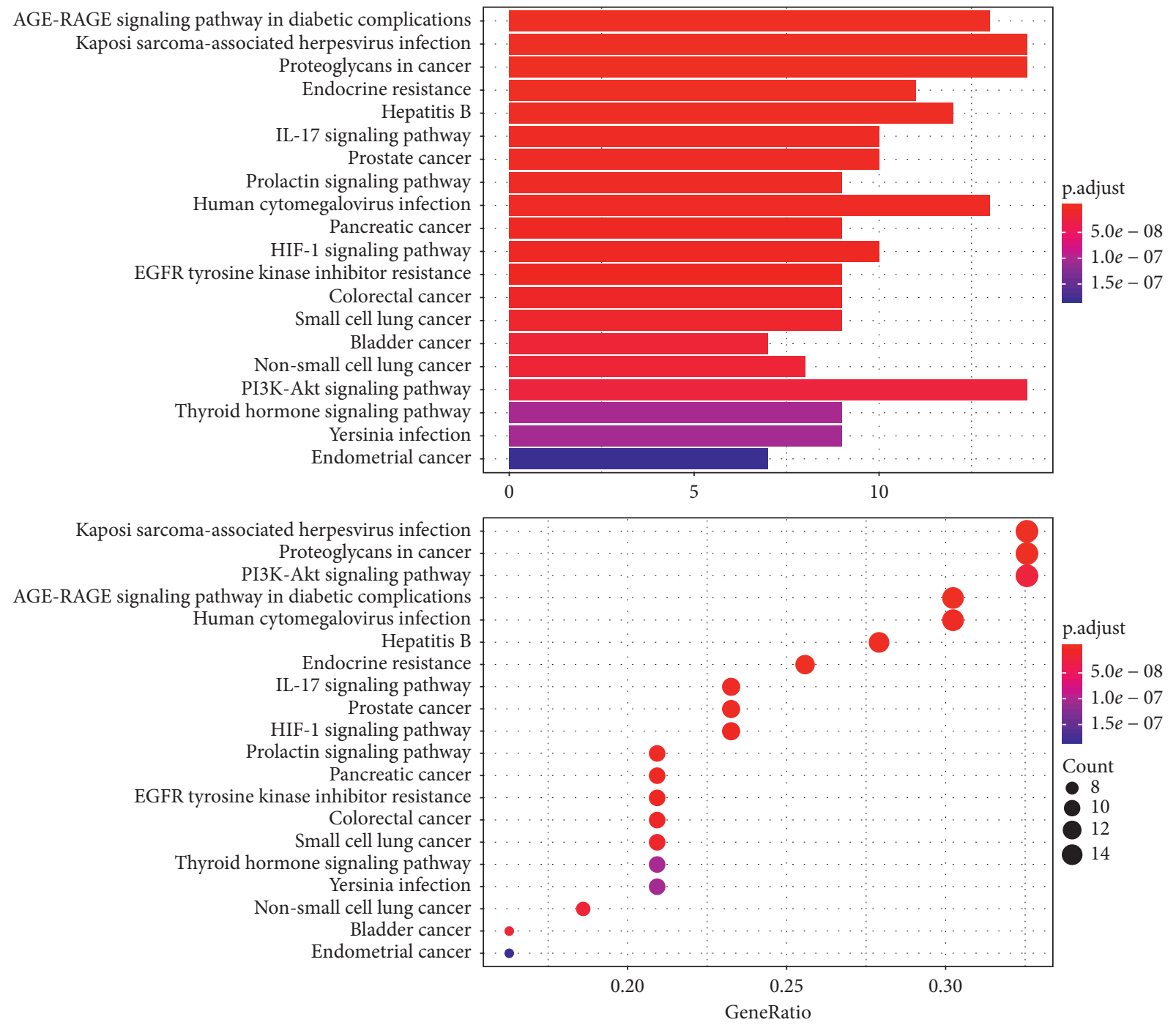

FIGURE 9: KEGG enrichment pathway analysis of therapeutic targets of CDD in treating PCOS.

[56]. In this study, several network pharmacology-based methods were used to predict potential targets. This approach provides new clues to exploring ethnopharmacology and herbal or even TCM formulas. Through the analysis of the putative therapeutic target network and biological functions in this study, the potential pharmacological and molecular mechanisms of CDD in treating PCOS were preliminary revealed.

In this study, the preliminary analysis based on network pharmacology provided a basis for subsequent studies on the pharmacodynamic ingredients and mechanisms of CDD. However, due to the limitations of the screening conditions and the TCM database, this study also has some shortcomings. Although a large number of targets and pathways were obtained through network pharmacology screening, these results must be verified by subsequent pharmacological experiments. In this study, the active components of CDD and their molecular mechanisms in PCOS were predicted overall, and a variety of potential therapeutic targets were explored. Thus, this study lays a good theoretical foundation for the next step of experimental verification and provides directions for further research on the molecular mechanism of PCOS.

\section{Data Availability}

The data used to support the findings of this study are available from the corresponding author upon request.

\section{Disclosure}

The funders had no role in study design, data collection and analysis, decision to publish, or preparation of the manuscript.

\section{Conflicts of Interest}

The authors declare that they have no conflicts of interest.

\section{Authors' Contributions}

XWT designed the study, analyzed the data, and wrote the manuscript; WLH supervised the study and revised the 
manuscript; TMY and WJH obtained and analyzed the data. All authors have read and agreed to publish this manuscript.

\section{Acknowledgments}

This work was supported by the Science and Technology Development Plan of SuZhou in 2018 (SYSD2018002), the Science and Technology Development Plan of SuZhou in 2018 (SYSD2018195), the Projects for Science and Technology of Chinese Medicine of Jiangsu Province in 2019 (YB201959), and the "333" Project in Jiangsu Province in 2019 (BRA2019140). The special funds of the Key Clinical Disease in Diagnosis and Treatment Technology of SuZhou in 2019 (LCZX201920) also provided financial support for this study.

\section{References}

[1] P. Spritzer, C. Barone, and F. Oliveira, "Hirsutism in polycystic ovary syndrome: pathophysiology and management," Current Pharmaceutical Design, vol. 22, no. 36, pp. 5603-5613, 2016.

[2] A. Nandi, Z. Chen, R. Patel, and L. Poretsky, "Polycystic ovary syndrome," Endocrinology and Metabolism Clinics of North America, vol. 43, no. 1, pp. 123-147, 2014.

[3] M. P. Lauritsen, J. G. Bentzen, A. Pinborg et al., "The prevalence of polycystic ovary syndrome in a normal population according to the Rotterdam criteria versus revised criteria including anti-mullerian hormone," Human Reproduction, vol. 29, no. 4, pp. 791-801, 2014.

[4] R. S. Legro, W. C. Dodson, P. M. Kris-Etherton et al., "Randomized controlled trial of preconception interventions in infertile women with polycystic ovary syndrome," The Journal of Clinical Endocrinology \& Metabolism, vol. 100, no. 11, pp. 4048-4058, 2015.

[5] R. S. Sarwer, "Polycystic ovary syndrome and cardiovascular disease: a premature association?" Endocrine Reviews, vol. 24, no. 3, pp. 302-312, 2003.

[6] R. L. Rosenfield and D. A. Ehrmann, "The pathogenesis of polycystic ovary syndrome (PCOS): the hypothesis of PCOS as functional ovarian hyperandrogenism revisited," Endocrine Reviews, vol. 37, no. 5, pp. 467-520, 2016.

[7] D. A. Dumesic, S. E. Oberfield, E. Stener-Victorin, J. C. Marshall, J. S. Laven, and R. S. Legro, "Scientific statement on the diagnostic criteria, epidemiology, pathophysiology, and molecular genetics of polycystic ovary syndrome," Endocrine Reviews, vol. 36, no. 5, pp. 487-525, 2015.

[8] H. F. Escobar-Morreale, M. Luque-Ramírez, and J. L. San Millán, "The molecular-genetic basis of functional hyperandrogenism and the polycystic ovary syndrome," Endocrine Reviews, vol. 26, no. 2, pp. 251-282, 2005.

[9] R. Wang, W. Li, E. M. Bordewijk et al., "First-line ovulation induction for polycystic ovary syndrome: an individual participant data meta-analysis," Human Reproduction Update, vol. 25, no. 6, pp. 717-732, 2019.

[10] A. H. Moll, L. C. Morley, M. Misso et al., "The management of anovulatory infertility in women with polycystic ovary syndrome: an analysis of the evidence to support the development of global WHO guidance," Human Reproduction Update, vol. 22, no. 6, pp. 687-708, 2016.

[11] C. R. McCartney and J. C. Marshall, "Polycystic ovary syndrome," New England Journal of Medicine, vol. 375, no. 1, pp. 54-64, 2016.
[12] S. Manzoor, M. A. Ganie, S. Amin et al., "Oral contraceptive use increases risk of inflammatory and coagulatory disorders in women with Polycystic Ovarian Syndrome: an observational study," Scientific Reports, vol. 9, no. 1, Article ID 10182, 2019.

[13] C.-H. Chen, H.-Y. Chin, H.-H. Chen, H.-Y. Chang, and W.-M. Liu, "Pills-related severe adverse events: a case report in Taiwan," Taiwanese Journal of Obstetrics and Gynecology, vol. 55, no. 4, pp. 588-590, 2016.

[14] R. A. Al Khalifah, I. D. Florez, B. Dennis, L. Thabane, and E. Bassilious, "Metformin or oral contraceptives for adolescents with polycystic ovarian syndrome: a meta-analysis," Pediatrics, vol. 137, no. 5, 2016.

[15] H. J. Teede, M. L. Misso, M. F. Costello et al., "Erratum. Recommendations from the international evidence-based guideline for the assessment and management of polycystic ovary syndrome," Human Reproduction, vol. 34, no. 2, p. 388, 2019.

[16] J. C. Lee, S. C. Pak, S. H. Lee et al., "The effect of herbal medicine on nerve growth factor in estradiol valerate-induced polycystic ovaries in rats," The American Journal of Chinese Medicine, vol. 31, no. 6, pp. 885-895, 2003.

[17] H. R. Choi, J. H. Kim, D. Lee, and H. G. Jo, "Cangfu daotan decoction for polycystic ovary syndrome: a protocol of systematic review and meta-analysis," Medicine, vol. 98, no. 39, Article ID e17321, 2019.

[18] C. F. Ding, C. Y. Wang, X. Yang, R. H. Zheng, Z. Z. Yan, and W. Q. Chen, "Effect of modified cangfu daotan decoction in improving endometrial receptivity in infertility patients with polycystic ovarian syndrome," Chinese Journal of Integrated Traditional and Western Medicine, vol. 34, no. 11, pp. 12971301, 2014.

[19] S. I. Berger and R. Iyengar, "Network analyses in systems pharmacology," Bioinformatics, vol. 25, no. 19, pp. 2466-2472, 2009.

[20] J. Ru, P. Li, J. Wang et al., "TCMSP: a database of systems pharmacology for drug discovery from herbal medicines," Journal of Cheminformatics, vol. 6, p. 13, 2014.

[21] S. Wang, Y. Tong, T. B. Ng et al., "Network pharmacological identification of active compounds and potential actions of Erxian decoction in alleviating menopause-related symptoms," Chinese Medicine, vol. 10, no. 1, 2015.

[22] K. X. Hu, X. Duan, L. Z. Han, H. Y. Ju, B. Wang, and Z. S. Tang, "Exploring pharmacological mechanisms of Xiang Ju tablets in the treatment of allergic rhinitis via a network pharmacology approach," Evidence-Based Complementary and Alternative Medicine, vol. 2019, Article ID 6272073, 13 pages, 2019.

[23] J. Huang, H. Tang, S. Cao, Y. He, and Y. Feng, "Molecular targets and associated potential pathways of danlu capsules in hyperplasia of mammary glands based on systems pharmacology," Evidence-Based Complementary and Alternative Medicine, vol. 2017, Article ID 1930598, 10 pages, 2017.

[24] W. Y. Lee, C. Y. Lee, Y. S. Kim, and C. E. Kim, "The methodological trends of traditional herbal medicine employing network pharmacology," Biomolecules, vol. 9, no. 8, 2019.

[25] S. Y. K. Fong, A. Bauer-Brandl, and M. Brandl, "Oral bioavailability enhancement through supersaturation: an update and meta-analysis," Expert Opinion on Drug Delivery, vol. 14, no. 3, pp. 403-426, 2017.

[26] D. Machado, M. Girardini, M. Viveiros, and M. Pieroni, "Challenging the drug-likeness dogma for new drug discovery 
in tuberculosis," Frontiers in Microbiology, vol. 9, p. 1367, 2018.

[27] Z. Ma, N. Wang, H. He, and X. Tang, "Pharmaceutical strategies of improving oral systemic bioavailability of curcumin for clinical application," Journal of Controlled Release, vol. 316, pp. 359-380, 2019.

[28] P. Artursson, K. Palm, and K. Luthman, "Caco-2 monolayers in experimental and theoretical predictions of drug transport," Advanced Drug Delivery Reviews, vol. 46, no. 1-3, pp. 27-43, 2001.

[29] M. Rebhan, V. Chalifa-Caspi, and J. Prilusky, "GeneCards: integrating information about genes, proteins and diseases," Trends in Genetics, vol. 13, no. 4, p. 163, 1997.

[30] A. Hamosh, A. F. Scott, J. Amberger, C. Bocchini, D. Valle, and V. A. McKusick, "Online Mendelian Inheritance in Man (OMIM), a knowledgebase of human genes and genetic disorders," Nucleic Acids Research, vol. 30, no. 1, pp. 52-55, 2002.

[31] R. Azziz, "Polycystic ovary syndrome," Obstetrics \& Gynecology, vol. 132, no. 2, pp. 321-336, 2018.

[32] S. Nekoonam, M. Naji, M. S. Nashtaei et al., "Expression of AKT1 along with AKT2 in granulosa-lutein cells of hyperandrogenic PCOS patients," Archives of Gynecology and Obstetrics, vol. 295, no. 4, pp. 1041-1050, 2017.

[33] D.-e.-S. Ali, M. Shah, A. Ali et al., "Treatment with metformin and combination of metformin plus pioglitazone on serum levels of IL-6 and IL- 8 in polycystic ovary syndrome: a randomized clinical trial," Hormone and Metabolic Research, vol. 51, no. 11, pp. 714-722, 2019.

[34] A. Ben Salem, F. Megdich, O. Kacem et al., "Vascular endothelial growth factor (VEGFA) gene variation in polycystic ovary syndrome in a Tunisian women population," $B M C$ Genomics, vol. 17, no. Suppl 9, p. 748, 2016.

[35] T. P. Ciaraldi, V. Aroda, S. R. Mudaliar, and R. R. Henry, "Inflammatory cytokines and chemokines, skeletal muscle and polycystic ovary syndrome: effects of pioglitazone and metformin treatment," Metabolism, vol. 62, no. 11, pp. 1587-1596, 2013.

[36] E. Diamanti-Kandarakis and A. Dunaif, "Insulin resistance and the polycystic ovary syndrome revisited: an update on mechanisms and implications," Endocrine Reviews, vol. 33, no. 6, pp. 981-1030, 2012.

[37] X. Jiao, W. Chen, J. Zhang et al., "Variant alleles of the ESR1, PPARG, HMGA2, and MTHFR genes are associated with polycystic ovary syndrome risk in a Chinese population: a case-control study," Frontiers in Endocrinology, vol. 9, p. 504, 2018.

[38] J. Ranjbaran, M. Farimani, H. Tavilani et al., "Matrix metalloproteinases 2 and 9 and MMP9/NGAL complex activity in women with PCOS," Reproduction, vol. 151, no. 4, pp. 305$311,2016$.

[39] A. S. Kelley, M. Puttabyatappa, J. N. Ciarelli et al., "Prenatal testosterone excess disrupts placental function in a sheep model of polycystic ovary syndrome," Endocrinology, vol. 160, no. 11, pp. 2663-2672, 2019.

[40] F. Wang, Z. Zhang, Z. Wang et al., "Expression and clinical significance of the HIF-1a/ET-2 signaling pathway during the development and treatment of polycystic ovary syndrome," Journal of Molecular Histology, vol. 46, no. 2, pp. 173-181, 2015.

[41] Q. Xie and X. Xiong, "Mesenchymal stem cells alleviate DHEA-Induced polycystic ovary syndrome (PCOS) by inhibiting inflammation in mice," Stem Cells International, vol. 2019, Article ID 9782373, 12 pages, 2019.
[42] Ö. Özçaka, N. Buduneli, B. O. Ceyhan et al., "Is interleukin-17 involved in the interaction between polycystic ovary syndrome and gingival inflammation?" Journal of Periodontology, vol. 84, no. 12, pp. 1827-1837, 2013.

[43] F. Foroozanfard, A. Soleimani, E. Arbab, M. Samimi, and M. R. Tamadon, "Relationship between IL-17 serum level and ambulatory blood pressure in women with polycystic ovary syndrome," Journal of Nephropathology, vol. 6, no. 6, pp. 15-24, 2017.

[44] A. M. Kay and C. L. Simpson, "The role of AGE/RAGE signaling in diabetes-mediated vascular calcification," Journal of Diabetes Research, vol. 2016, Article ID 6809703, 8 pages, 2016.

[45] Z. Merhi, "Advanced glycation end products and their relevance in female reproduction," Human Reproduction, vol. 29, no. 1, pp. 135-145, 2014.

[46] C. Delcour, G. Robin, J. Young, and D. Dewailly, "PCOS and Hyperprolactinemia: what do we know in 2019?" Clinical Medicine Insights: Reproductive Health, vol. 13, Article ID 1179558119871921, 2019.

[47] Y. Zhang, F. Meng, X. Sun et al., "Hyperandrogenism and insulin resistance contribute to hepatic steatosis and inflammation in female rat liver," Oncotarget, vol. 9, no. 26, pp. 18180-18197, 2018.

[48] W.-F. Yeung, K.-F. Chung, K.-Y. Ng, Y.-M. Yu, E. T.-C. Ziea, and B. F.-L. Ng, "A systematic review on the efficacy, safety and types of Chinese herbal medicine for depression," Journal of Psychiatric Research, vol. 57, pp. 165-175, 2014.

[49] W.-z. Yang, Y. Hu, W.-y. Wu, M. Ye, and D.-a. Guo, "Saponins in the genus Panax L. (Araliaceae): a systematic review of their chemical diversity," Phytochemistry, vol. 106, pp. 724, 2014.

[50] R. Wang, Q. Sun, F. Wang et al., "Efficacy and safety of Chinese herbal medicine on ovarian cancer after reduction surgery and adjuvant chemotherapy: a systematic review and meta-analysis," Frontiers in Oncology, vol. 9, p. 730, 2019.

[51] Y. Wang, X.-T. Lou, Y.-H. Shi, Q. Tong, and G.-Q. Zheng, "Erxian decoction, a Chinese herbal formula, for menopausal syndrome: an updated systematic review," Journal of Ethnopharmacology, vol. 234, pp. 8-20, 2019.

[52] J. V. Franco, T. Turk, J. H. Jung et al., "Pharmacological interventions for treating chronic prostatitis/chronic pelvic pain syndrome," The Cochrane Database of Systematic Reviews, vol. 10, no. 10, p. Cd012552, 2019.

[53] Y. Jiang, N. Liu, S. Zhu, X. Hu, D. Chang, and J. Liu, "Elucidation of the mechanisms and molecular targets of yiqi shexue formula for treatment of primary immune thrombocytopenia based on network pharmacology," Frontiers in Pharmacology, vol. 10, p. 1136, 2019.

[54] X. D. Miao, L. J. Zheng, Z. Z. Zhao et al., "Protective effect and mechanism of boswellic acid and myrrha sesquiterpenes with different proportions of compatibility on neuroinflammation by LPS-induced BV2 cells combined with network pharmacology," Molecules (Basel, Switzerland), vol. 24, no. 21, 2019.

[55] Q. Guo, W. Niu, X. Li et al., "Study on hypoglycemic effect of the drug pair of Astragalus radix and dioscoreae rhizoma in T2DM rats by network pharmacology and metabonomics," Molecules (Basel, Switzerland), vol. 24, no. 22, 2019.

[56] M. J. Lin, H. W. Chen, P. H. Liu, W. J. Cheng, S. L. Kuo, and M. C. Kao, "The prescription patterns of traditional Chinese medicine for women with polycystic ovary syndrome in Taiwan: a nationwide population-based study," Medicine, vol. 98, no. 24, Article ID e15890, 2019. 